\section{Use of Gardening Programs as an Intervention to Increase Children's Visual-motor Integration}

\author{
Aime Sommerfeld ${ }^{1}$, Amy McFarland ${ }^{2}$, Tina M. Waliczek ${ }^{3}$, and \\ Jayne Zajicek ${ }^{4}$
}

AdDITIONAL INDEX WORDS. Beery-Buktenica visual-motor integration, childhood development, horticultural therapy, motor skills, school gardens

Summary. Visual-motor integration is influential in childhood development. Historical anecdotal evidence supports gardening as aiding in children's development of fine and gross motor skills. The main objective of this study was to examine the effect of a school gardening program on children's development of visual-motor integration. Preschool children ages 2 to 6 years old enrolled in private tuition-based schools were included in the sample. For 6 months, control group students studied using a traditional school curriculum whereas treatment group students participated in gardening as part of their lessons. The BeeryBuktenica visual-motor integration short-form instrument was used to quantitatively measure students' levels of visual-motor integration. No significant differences were found in overall comparisons between the treatment and control group students. However, in demographic comparisons, significance was found; standardized scores for males in the treatment group improved whereas scores for males in the control group decreased. Results indicated that male preschoolers may respond especially well to gardening programs in the classroom in developing visual-motor integration.

M odern conveniences and technologies providing instant gratification are often the same, offering adults and children opportunities for indoor-based entertainment and screen-time activities (Pergams and Zaradic, 2006; Renard, 2005 ) that may restrict motor skill development in children. Pergams and Zaradic (2006) define their new term videophilia, as "the new human tendency to focus on sedentary activities involving electronic media." Youth motor skills previously developed through outdoor activities and

Received for publication 4 May 2021. Accepted for publication 23 June 2021.

Published online 4 August 2021

${ }^{1}$ Waco Family Medicine, College Station, TX 77843

${ }^{2}$ Frederik Meijer Honors College, Grand Valley State University, Allendale, MI 49401

${ }^{3}$ Department of Agricultural Sciences, Texas State University, San Marcos, TX 78666

${ }^{4}$ Department of Horticultural Sciences, Texas A\&M University, College Station, TX 77843

A.S. is an Urban Garden/Agriculture Consultant.

A.M. is an Associate Professor

T.M.W. is a Professor of Horticulture.

T.M.W. is the corresponding author. E-mail: tc10@ txstate.edu.

This is an open access article distributed under the CC BY-NC-ND license (https://creativecommons. org/licenses/by-nc-nd/4.0/).

https://doi.org/10.21273/HORTTECH04887-21 interactions with nature have been replaced with the use of hand-held electronic gaming devices and buttons on screens (Straker et al., 2011). The 2.5to 6-year age range is often referred to as the early childhood development stage or the Piagetian preoperational stage (Siegler et al., 2006). Early childhood is a time for rapid growth in areas of $\operatorname{cog}$ nitive, emotional, physical, and social development (DeHart et al., 2004). During this stage there is great fluctuation in a child's capacity for growth and development, where much depends on everyday interactions and opportunities (DeHart et al., 2004). Research suggests natural outdoor environments offer youth greater capacity for physical activity, increasing opportunities for gross motor skill development, although not excluding development of fine motor skills (Baker et al., 2015; Fjortoft, 2001; Straker et al., 2011).

Although all motor skills are important, Sortor and Kulp (2003) propose a significant link between visual perceptual skill development and math and reading achievement in elementary school students. Hand-eye coordination is a commonly used term to encompass a targeted concept, whereas visual-motor integration is defined by Beery and Beery (2006) as, "the degree to which visual perception and finger- hand movements are well coordinated." According to a study examining the relationship between visual-motor coordination, hand-eye coordination, and the quality of handwriting, Kaiser et al. (2009) reflected that, although similar, the terms visual-motor integration and hand-eye coordination are slightly different in that hand-eye coordination needs more visual control (e.g., to trace items) whereas visual-motor integration requires more visual perception (e.g., copying forms). Fine motor skills can be defined as the coordination of muscles, bones, and nerves to produce small, precise movements.

The U.S. National Library of Medicine (2014) maintains that children develop fine motor skills over time through a combination of repetition and teaching. To acquire fine motor control, children must have awareness and planning abilities, adequate coordination and muscle strength, as well as normal sensation. Proper maturation of the nervous system in conjunction with these variables allows development of fine motor abilities, including examples such as controlled scissor work, writing and drawing tasks, folding and stacking abilities, and zipper control (U.S. National Library of Medicine, 2014).

Visual-motor integration as a fine motor skill has been linked to academic success in fields such as handwriting, math, and reading (Grissmer et al., 2010; Lahav et al., 2013; Sortor and Kulp, 2003), as well as emotional health and self-esteem (Bart et al., 2007; Lahav et al., 2013). Grissmer et al. (2010) conducted a study examining data from three longitudinal data sets with motor function measurements and found that fine motor skills were a strong and consistent predictor of later achievement in academic settings. In a study investigating academic achievement in relation to performance on a visual-motor integration measurement, Sortor and Kulp (2003) concluded visual perceptual skills are factors related significantly to achievement in math and reading. Rule and Stewart (2002) reported the nature of fine motor skill activities along with the way children were instructed to complete them were important factors in increased performance. Their study reported teachers' notice of students' enjoyment in specified activities were outside of traditional fine motor skill development work, and 
children spoke with enthusiasm about using their imaginations and addressing the challenging work (Rule and Stewart, 2002).

Deficiencies in visual-motor integration can attribute to a delay of basic learning skills associated with normal educational development (Sortor and Kulp, 2003). Lahav et al. (2013) reported that self-esteem and social performance may be tied to motor difficulties, including visual-motor skills, and stated that, "the association between motor function and emotional status is well established and improvement of motor function can promote emotional health." In a study looking at the predictive abilities of kindergarten motor scores on later school adjustment, Bart et al. (2007) reported academic and social failures caused by motor inadequacies may cause a child to lose selfconfidence, and become anxious and withdrawn at school. Bart et al. (2007) also postulated poor visual-motor measures could be related to increased frustration, difficulty sustaining attention, and poor overall achievement, supporting tendencies for disruptive behaviors in the classroom.

The famous educator Maria Montessori believed children must exercise their "various coordinated movements" (her term for motor skills), and realized the garden is an ideal environment for this to take place (Gutek, 2004). Montessori understood gardening and its related chores can improve motor development by allowing children to manipulate various garden implements; digging, carrying, sorting, harvesting, and weeding all require fine and gross movements (Gutek, 2004). Investigations on the effect of gardening on physical fitness variables has been studied to some degree, with research showing that gardening is a suitable low- to moderate-intensity exercise for most people (Park et al., 2008,2015 ). However, studies regarding gardening and its impact on motor skills in children is limited (Baker et al., 2015). The purpose of this study was to examine the effect of a school gardening program on children's development of visual-motor integration.

\section{Materials and methods}

Research Design. Quasi-experimental nonequivalent group design was used for this study. This design was selected because campus populations were kept intact and thus were not available for random assignment.

SAMPLE. The population for this study was preschool children ranging in age from 2 to 6 years. The sample was drawn from schools that had no active garden onsite in the past year, but were interested in constructing a garden for educational opportunities. Treatment and control schools were selected with comparable school atmospheres and student demographics. Control group schools were matched as closely as possible to the treatment group based on teaching methodologies, age of children served, and interest in a garden program upon study completion. The interest in a garden program was important to ensure alignment of teaching interests between control and treatment groups. All schools selected for the study were private tuition-based preschool programs.

This study included three different class levels in testing, but focused on what would be equivalent to the traditional 3- and 4-year-old prekindergarten classrooms. However, it is important to note that because of the progression methods in this school, there is a mix of ages in each classroom situation. There was one class of Transition or prekindergarten 3, which consisted of 12 students, and two rooms of prekindergarten 4 , one with 12 students and the other with 14 students at the beginning of the study. Every student in the treatment school that had a class involved with garden activities received consent forms to take home that contained a description of the study and a seed packet as an incentive for returning the signed permission forms. Other incentives for the treatment school included supplies to build and establish a garden onsite, the researcher as a contact person for the duration of the study, and bimonthly lessons/activities carried out by graduate students.

Two control schools were used for this study. These control groups agreed to abstain from participating in garden activities until completion of the study, and they continued with their previously established curriculum. Control school A enrolled 12 students at the time of testing and was conducted more along the lines of what would be expected in a public school setting, with more focus on work and less on free play. Control school B was much larger than the other two and only certain teachers opted to be involved in this study. Each class was divided by year and consisted of about 15 students, with two to three classrooms per age division. Incentives of seed packets were sent home with each student in consent form packages that included a description of the study. The control schools were offered $\$ 500$ for participation in the study to be used for garden supplies or garden-related materials upon completion of research measures.

In addition to parental permission, the researcher required participant consent during each assessment measure. If at any point the participants (children) were uncomfortable or unwilling to take part in any assessment, or demonstrated unease, they were asked whether they would like to continue or to be "all done/finished." All testing procedures were approved by the institutional review board before study initiation.

Garden TREATMENT PROGRAM. A garden was built at the treatment site through a coordinated effort between school administrators, researchers, and school families, including the children. The enclosed garden was located next to the large playground, separated by a chain link fence and unlocked gate. The school garden consisted of a series of raised beds of different sizes to maximize materials on hand, and to create areas where children could work and reach easily. Raised beds included six that were $\approx 4 \times 6 \mathrm{ft}(1.2 \times 1.8 \mathrm{~m})$ and three that were $2 \times 2 \mathrm{ft}(0.6 \times 0.6 \mathrm{~m})$. Two trellis systems near the fence had small beds around them, and a teepee structure was constructed using bamboo and cinder blocks.

The garden was planted primarily with annual vegetable crops and occasionally annual flowers. Efforts were made to plant both seeds and transplants, a variety of edible plant parts, as well as short- and longer season crops to offer a range of experiences for the students.

Researchers worked with children in the garden at least every other week doing basic activities such as watering, planting, harvesting, and pulling weeds. Once per month, a larger activity was conducted around a lesson including topics such as plant life cycles, nutrition, and vegetable tastetesting. Teachers were encouraged to use the garden as a teaching tool 
outside of visits by the researchers; however, use of the garden was not required.

Instrumentation. Pre- and posttests were used to measure student hand-eye coordination. The researcher administered both pre- and posttests at treatment and control schools with a testing interval of less than 6 months to control for maturation effects. Maturation effects refer to the normal development of a child, and, according to Gay et al. (2006), 6 months is an appropriate time frame to control for this threat to internal validity.

Visual-motor integration was evaluated using the Beery-Buktenica developmental test of visual-motor integration [Beery VMI (Beery and Beery, 2006)]. The Beery VMI was developed initially in 1967 to measure children's visual-motor integration. The test asks participants to imitate or copy a developmental sequence of geometric forms using the provided booklet and pencil, or black ballpoint pen, to examine visual-motor integration. Multiple forms of the Beery VMI are available and used for various groups, but the selected short form is used for children 2 to 7 years of age and consists of 21 items. Researchers have found the Beery VMI to be a reliable predictor of achievement, especially in lower grade levels, before youth learn to compensate for learning deficiencies, with an overall reliability of 0.92 (Beery and Beery, 2006). Content validity, the degree to which a test measures its intended content area (Gay et al., 2006), was reported at 0.96. Geometric forms are used as opposed to alphabetic or numeric forms to avoid gender, socioeconomic, and cultural barriers.

Instructions for the Beery VMI are fairly straightforward (Beery and Beery, 2006). For our study, the researcher sat down with each student individually talking him or her through the procedure. A number-two pencil was used, and erasing was not allowed. Students were encouraged to keep the booklet straight and square in front of them while working. The researcher instructed the students to do the best they could in copying each item and observed students working to ensure that no sections of the test were skipped.

All participants, regardless of age, began with the imitation exercise. The imitation exercise called for the participant to copy (imitate) the researcher for the first three geometric forms in the testing booklet. When those forms were completed, the participants advanced to the portion of the booklet where geometric shapes were provided for unassisted visual copying. If participants were unable to complete the imitation portion, they were directed to the booklet's blank boxes used for the marking and scribbling portion of assessment. The marking and scribbling assessment allowed the participants the freedom to mark on the page without following specific rules or guidelines. After markings were made, participants were allowed to return to the imitation portion of the test booklet. As dictated by the Beery VMI testing administration guide, participants were allowed to work as far in the testing booklet as they wanted. Although participants were asked if they would like to continue working or be "finished with this work" after copying three consecutive figures incorrectly, most enjoyed the activity and chose to continue working.

Scoring. Visual-motor integration data were scored using procedures developed by Beery and Beery (2006). Pre- and posttest booklets from all schools were combined and shuffled for random scoring to reduce possible bias.

Raw scores ranged from 0 to 20 and were transformed to standardized scores using scoring criteria dictated in the instruction manual. Standard scores for Beery VMI have a mean of 100 , with an SD of 15 for all age groups, and are based on means for raw score distributions. Scores are age specific down to 2 months, with $15 \mathrm{~d}$ or more in the birthdate month rounded to a higher month.

Scoring for the Beery VMI is based on score vs. no score criteria, where 1 point is awarded for each imitated or correctly copied item and up to three consecutive failures. Each geometric figure must meet criteria such as participant's first attempt, internal and/or external angle requirement, length of lines, correct axis, and continuity of pencil strokes. If there was uncertainty of a form meeting criteria, the form was scored as criteria met and a point was awarded. Sometimes participants realized they were not copying a form correctly and made multiple attempts. In this situation, the first attempt was identified and scored using context clues from other geometric forms or test administrator notations. The test manual was used heavily as a resource because it provided multiple-score and no-score examples for each geometric form, and descriptive text emphasizing form requirements (Beery and Beery, 2006).

The researcher who administered the test booklets as well as a secondary researcher scored instruments to check interrater reliability on standardized scores. Interrater reliability was assessed using a two-way mixed model, consistency type, average measures intraclass correlation (ICC) (Hallgren, 2012). The ICC $(3,2)$ analysis revealed a correlation in the excellent range- $\mathrm{ICC}=$ 0.955 (Cicchetti, 1994; Hallgren, 2012)_indicating a high degree of agreement between visual-motor integration scorers for this study. The standardized scores from the two researchers were then combined and averaged to use in data analysis.

Data analysis. Raw data were entered into a spreadsheet (Microsoft Excel; Microsoft Corp., Redmond, WA) for reference and data transformation, then transferred and analyzed using statistical software (IBM SPSS Statistics version 22.0; IBM Corp., Armonk, NY). Statistical procedures included descriptive statistics, frequencies when applicable, and analysis of variance tests to determine differences between scores of pretests and posttests for treatment and control groups.

\section{Results and discussion}

Demographics. The study gathered 88 participant scores during the pretest section of the study and 81 scores at posttest, resulting in 81 possible scores for analysis. Age and sample number breakdowns for control and treatment groups are provided in Table 1. Although the treatment school age range contains the oldest participant in the groups, the larger SD demonstrates that average age in the control group is older than the treatment group by about 1 month. In the final analysis, the treatment school had a younger population compared with the control schools by more than 2.5 months. Furthermore, maturation effects, which are normal gains attributed to growth and development, posed a threat to internal validity (Tuckman, 1999). The threats 
Table 1. Age comparison of Beery-Buktenica visual-motor integration (Beery VMI) for the overall sample, treatment, and control groups during the pre- and posttest time periods in the study of the influence of a school garden program on children's visual-motor integration.

\begin{tabular}{lcccc}
\hline & Participants (no.) & Age range (yr) & Avg age (yr) & SD (d) \\
\hline Pretest score $^{\mathrm{z}}$ & & & & \\
$\quad$ Overall & 88 & $2.03-6.32$ & 4.07 & 339.38 \\
Treatment & 42 & $2.03-6.32$ & 3.73 & 361.01 \\
Control & 46 & $2.75-5.88$ & 4.38 & 279.03 \\
Posttest score & & & & \\
$\quad$ Overall & & $2.51-6.82$ & 4.48 & 336.30 \\
Treatment & 41 & $2.51-6.82$ & 4.22 & 363.68 \\
Control & 40 & $3.12-6.25$ & 4.72 & 283.91 \\
\hline
\end{tabular}

${ }^{\mathrm{z}}$ Standard scores on the Beery VMI have a mean of 100 with an SD of 15 for all age groups and are based on the means of raw score distributions (Beery and Beery, 2006).

of maturation effects are reduced with a shorter testing period, so validity was controlled for by using a 6-month pre-/posttest time frame, which was consistent between the control and treatment groups (Gay et al., 2006). The Beery VMI also controls for effects of age through scoring procedures (Beery and Beery, 2006). Beery VMI scores are age specific to within 2 months. The consistent procedures regarding the pre-/posttest time frame ensured that any maturation effects were experienced similarly between the control and treatment groups, further reducing the impact on our study.

STATISTICAL ANALYSIS FOR BeEry VMI. A split-plot analysis of variance (mixed design) compared the change in scores between a pre- and posttest measure in regard to treatment and control schools. No significant main effect $(\mathrm{F}=1.764, P=0.188)$ was found when comparing the treatment and control schools regarding the change in visual-motor integration scores from pre- to posttest (Table 2). Because of the age-specific standardized scoring procedures for the visualmotor integration, any change in scores is notable. In the instance that a group maintained the same average score from pre- to posttest, the interpretation is that the group improved their visual-motor integration at a constant rate reflective of their age. A constant score of 100 reflects age-appropriate development and systematic improvements of visual-motor integration consistent with the aging process (Beery and Beery, 2006).

Although there was no significant difference between the treatment and control school averages, it may be worth noting that descriptive statistics indicated the treatment school scores showed an improvement of 1.75 , meaning the treatment group raised its average score to the Beery VMI standardized average of 100 . The control school scores decreased by 2.27 points, meaning that although the control schools scores were still above average, student visual-motor integration did not maintain consistent ability levels. Because control school scores were above a standardized average, it could be that it was not possible to maintain an elevated score because scores will trend toward the standardized average as students age. Results of this study are similar to a larger scale study in a public urban school district in which the effect of an extension garden curriculum on visual-motor integration was explored in preschool and kindergarten students (Baker et al., 2015). Measurement was taken with the Beery VMI short form, and

Table 2. Average of Beery-Buktenica visual-motor integration (Beery VMI) preand posttest standardized scores by treatment and control group in the study examining the influence of a school garden program on children's visual-motor integration.

\begin{tabular}{lccccc}
\hline & Participants (no.) & Pretest score $^{\mathrm{z}}$ & Posttest score $^{\mathrm{z}}$ & F value & $\boldsymbol{P}_{\text {value }}$ \\
\hline Treatment & 39 & 98.62 & 100.37 & 1.764 & 0.188 \\
Control & 40 & 110.14 & 107.87 & & \\
\hline
\end{tabular}

${ }^{\mathrm{z}}$ Standard scores on the Beery VMI have a mean of 100 with an SD of 15 for all age groups and are based on the means of raw score distributions (Beery and Beery, 2006). control group scores were reported to decrease whereas treatment group scores maintained a level that reflected consistent capabilities (Baker et al., 2015). Similarities between the smaller and larger scale studies are important because they demonstrate strength with the consistent findings, even if the findings are not significant.

When exploring gender and Beery VMI in treatment and control schools through a split-plot analysis of variance, no significant differences were found in pre- vs. posttest averages for females $(\mathrm{F}=0.010, P=0.919)$. However, significance was found in average scores for males $(\mathrm{F}=5.22, P=0.028)$, meaning the change in score from pretest to posttest was statistically significant between the control and treatment groups. Standardized scores for males in the treatment group showed substantial improvement, whereas scores for males at the control schools decreased (Table 3).

All groups pretesting above average reflected a decrease in scores for posttest measures. Scores for females in both the treatment and control schools decreased, reflecting a minimal change in scores and demonstrating a consistent aptitude for their age. Male scores in the control group decreased by an average of 3.06 points, whereas male treatment school scores increased by 9.17 points, approaching the $15-$ point SD for the measure. In addition, males in the control group showed the greatest decline in scores (3.06 points). Although the sample size is small, this trend could suggest that males may be more likely to respond to a physical activity such as gardening to learn motor skills such as visual-motor integration.

A review of the literature by Hinkley et al. (2008) concluded that, although physical activity behaviors of preschool children are multidimensional, males are typically more active than girls, and a study by Pate et al. (2004) found boys to participate in significantly more moderate-to-vigorous physical activity and vigorous physical activity compared with girls. Using these different physical activity levels to channel learning experiences may be a way to reach and teach a specific cohort. Gender results from this intervention support the recommendation of Bardid et al. (2013) that early motor skill programs implement, 
Table 3. Average of Beery-Buktenica visual-motor integration (Beery VMI) preand posttest standardized scores by gender and school in the study examining the influence of a school garden program on children's visual-motor integration.

\begin{tabular}{|c|c|c|c|c|c|c|c|}
\hline & $\begin{array}{l}\text { Participants } \\
\text { (no.) }\end{array}$ & $\begin{array}{l}\text { Pretest } \\
\text { score }^{\mathrm{z}}\end{array}$ & SD & $\begin{array}{l}\text { Posttest } \\
\text { score }^{\mathrm{z}}\end{array}$ & SD & $\begin{array}{c}\mathrm{F} \\
\text { value }\end{array}$ & $\begin{array}{c}P \\
\text { value }\end{array}$ \\
\hline Male & & & & & & 5.22 & $0.028 *$ \\
\hline Treatment & 12 & 88.96 & 17.83 & 98.13 & 14.40 & & \\
\hline Control & 23 & 108.28 & 15.07 & 105.22 & 11.71 & & \\
\hline Female & & & & & & 0.010 & 0.919 \\
\hline Treatment & 27 & 102.91 & 15.57 & 101.37 & 11.98 & & \\
\hline Control & 17 & 112.65 & 13.72 & 111.47 & 12.27 & & \\
\hline
\end{tabular}

${ }^{\mathrm{z}}$ Standard scores on the Beery VMI have a mean of 100 with an SD of 15 for all age groups and are based on the means of raw score distributions (Beery and Beery, 2006).

*Significant at $P \leq 0.05$.

or at least consider, gender-specific approaches. However, results from this study also reiterate the suggestion by Baker et al. (2015) that a gardening program may be an effective way to maintain a certain level of visualmotor integration capabilities.

\section{Conclusion}

The objective for this study was to determine whether participation in a school gardening program affected children's visual-motor integration, or the degree to which fine motor skills and visual perception are coordinated (Beery and Beery, 2006). One limitation of this study is that it was small in size. More research with larger sample sizes and diversity in sample participants is needed to understand more fully the relationship between gardening and visual-motor integration. Although no significant main effect was found when comparing treatment and control schools' change in visualmotor integration scores from pre- to posttests, when exploring gender, significance was found in average change in scores for males. Only males in the treatment group reported an increase in average scores on the visual-motor integration measure, from 88.96 to 98.13. Because scores for visual-motor integration are controlled for age, an increase in average score reveals growth beyond that typical for a specific person at a specific time. However, these scores are also standardized and, although they represent a significant increase, the increase simply brings them close to the standardized average of 100 points.

According to Rule and Stewart (2002), teachers should include carefully constructed and coached activities that focus on fine motor skill development and challenge students, but spark their imaginations as well. Although males in the treatment group for this study improved without constructed and coached fine motor activities, the elevated scores of the control schools that implemented directed activities for fine motor skill development support these structured lessons. However, if the garden intervention was combined with specific fine motor skill activities such as counting or separating seeds, it is feasible that the score would improve in overall treatment groups at a higher rate.

The importance of visual-motor integration has been demonstrated to influence a variety of academic achievements, including readiness in handwriting, as well as math, reading, and written expression (Carlson et al., 2013; Kaiser et al., 2009; Sortor and Kulp, 2003), making it an important steppingstone in early childhood education. A study by Carlson et al. (2013) emphasized that visual-motor coordination (fine motor control) does not appear to be related significantly to achievement, but visual-spatial integration (visual information processing integrating with fine motor control) is likely to be driving academic achievement. A meta-analysis of physical activity interventions for preschoolers found the most successful interventions to be "(a) provided in the early-learning environment, (b) directed by teachers, (c) to incorporate environmental changes, (d) to promote unstructured activities or free play, and (e) to provide outdoor play time" (Gordon et al., 2013). Although this research was examining physical activity, physical activity includes the use of fine and gross motor skills. An early-childhood garden program can provide all five attributes found in the meta-analysis for a successful preschool intervention program. Implementation of a garden program in an early-childhood education program allows an interesting way to provide visual-motor integration opportunities to all students, better preparing them for academic success in later years.

\section{Literature cited}

Baker, M., T.M. Waliczek, and J.M. Zajicek. 2015. The effect of school gardening activities on visual-motor integration of pre-school and kindergarten students. J. Therapeutic Res. 25:3-14.

Bardid, F., F. Deconinck, S. Descamps, L. Verhoeven, G. DePooter, M. Lenoir, and E. D'Hondt. 2013. The effectiveness of a fundamental motor skill intervention in pre-schoolers with motor problems depends on gender but not environmental context. Res. Dev. Disabil. 34:4571-4581, doi: 10.1016/j.ridd.2013.09.035.

Bart, O., D. Hajami, and Y. Bar-Haim. 2007. Predicting school adjustment from motor abilities in kindergarten. Infant Child Dev. 16:597-615, doi: 10.1002/ icd.514.

Beery, K. and N. Beery. 2006. The BeeryBuktenica developmental test of visualmotor integration: Administration, scoring, and teaching manual. 5th ed. (short form). NCS Pearson, Minneapolis, MN.

Carlson, A.G., E. Rowe, and T.W. Curby. 2013. Disentangling fine motor skills' relations to academic achievement: The relative contributions of visual-spatial integration and visual-motor coordination. J. Genet. Psychol. 174:514-533, doi: 10.1080/ 00221325.2012.717122.

Cicchetti, D.V. 1994. Guidelines, criteria, and rules of thumb for evaluating normed and standardized assessment instruments in psychology. Psychol. Assess. 6:284-290, doi: 10.1037/1040-3590.6.4.284.

DeHart, G.B., L.A. Srouf, and R.G. Cooper. 2004. Child development: Its nature and course. 5th ed. McGraw-Hill, New York, NY.

Fjortoft, I. 2001. The natural environment as a playground for children: The impact of outdoor play activities in pre-primary school children. Early Child. Educ. J. 29:111-117, doi: 10.1023/a:1012576913074.

Gay, L.R., G.E. Mills, and P. Airasian. 2006. Educational research: Competencies for analysis and application. Pearson Education, Upper Saddle River, NJ.

Gordon, E.S., P. Tucker, S.M. Burke, and A.V. Carron. 2013. Effectiveness of physical activity interventions for preschoolers: A meta-analysis. Res. Q. Exerc. Sport 84: 
287-294, doi: 10.1080/02701367.2013. 813894 .

Grissmer, D., K. Grimm, S. Aiyer, W. Murrah, and J. Steele. 2010. Fine motor skills and early comprehension of the world: Two new school readiness indicators. Dev. Psychol. 46:1008-1017, doi: 10.1037/ a0020104.

Gutek, G. 2004. The Montessori method: The origins of an educational innovation: Including an abridged and annotated edition of Maria Montessori's the Montessori method. Rowman and Littlefield Publishers, Lanham, MD.

Hallgren, K.A. 2012. Computing interrater reliability for observational data: An overview and tutorial. Tutor. Quant. Methods Psychol. 8:23-34, doi: 10.20 982/tqmp.08.1.p023.

Hinkley, T., D. Crawford, J. Salmon, A. Okely, and K. Hesketh. 2008. Preschool children and physical activity: A review of correlates. Amer. J. Prev. Med. 34:435441, doi: 10.1016/j.amepre.2008.02.001.

Kaiser, M., J. Albaret, and P. Doudin. 2009. Relationship between visual-motor integration, eye-hand coordination, and quality of handwriting. J. Occup. Ther. Sch. Early Interv. 2:87-95, doi: 10.1080/ 19411240903146228.
Lahav, O., A. Apter, and N. Ratzon. 2013. Psychological adjustment and levels of self-esteem in children with visual-motor integration difficulties influences the results of a randomized intervention trial. Res. Dev. Disabil. 34:56-64, doi: 10.1016/ j.ridd.2012.07.024.

Park, S., C. Shoemaker, and M. Haub. 2008. A preliminary investigation on exercise intensities of gardening tasks in older adults. Percept. Mot. Skills 107:974-980, doi: 10.2466/pms.107.3.974-980.

Park, S.A., H.S. Lee, K.S. Lee, K.C. Son, and C. Shoemaker. 2015. The metabolic costs of gardening tasks in children. HortTechnology 23:589-594, doi: 10.21273/ HORTTECH.23.5.589.

Pate, R., K. Pfeiffer, S. Trost, P. Ziegler, and M. Dowda. 2004. Physical activity among children attending preschools. Pediatrics 11:1258-1263, doi: 10.1542/ peds.2003-1088-L.

Pergams, O.R.W. and P.A. Zaradic. 2006. Is love of nature in the US becoming love of electronic media? J. Environ. Mgt. 80:387393, doi: 10.1016/j.jenvman.2006.02.001.

Renard, L. 2005. Teaching the DIG generation. Educ. Leadersh. 62:44.

Rule, A.C. and R.A. Stewart. 2002. Effects of practical life materials on kindergartners' fine motor skills. Early Child. Educ. J. 30:9-13, doi: 10.1023/A:1016533729704.

Siegler, R., J. DeLoache, and N. Eisenberg. 2006. How children develop. 2nd ed. Worth Publishing, New York, NY.

Sortor, J.M. and M.T. Kulp. 2003. Are the results of the Beery-Buktenica developmental test of visual-motor integration and its subtests related to achievement test scores? Optom. Vis. Sci. 80:758-763, doi: 10.1097/00006324-200311000-00 013 .

Straker, L.M., A.C. Campbell, L.M. Jensen, D.R. Metcalf, A.J. Smith, R.A. Abbott, C.M. Pollock, and J.P. Piek. 2011. Rationale, design, and methods for a randomized and controlled trial of the impact of virtual reality games on motor competence, physical activity, and mental health in children with developmental coordination disorder. BMC Public Health 11:654, doi: 10.1186/ 1471-2458-11-654.

Tuckman, B.W. 1999. Conducting educational research. 5th ed. Wadsworth Group, Belmont, CA.

U.S. National Library of Medicine. 2014. Fine motor control. 4 May 2021. <http://www.nlm.nih.gov/medlineplus/ ency/article/002364.htm $>$. 